\title{
Use of Traditional Chinese Medicine in the Management of Urinary Stone Disease
}

\author{
Ricardo Miyaoka, Manoj Monga \\ Department of Urologic Surgery, University of Minnesota, Minneapolis, Minnesota, USA
}

\begin{abstract}
Objective: To assess the evidence-based literature supporting the use of traditional Chinese medicine Kampo herbal and Acupuncture in stone disease management.

Materials and Methods: Four of the most commonly used herbal components of Kampo medicine in the treatment of stone disease are described according to their in vitro and in vivo effects. We also reviewed the role of Acupuncture in urologic clinical setting as well as its proposed mechanisms of action and results. Medline database was assessed using isolated and conjugated key words (Chinese Medicine, Kampo, Chinese Herbal, Calculi, Stone Disease, Kidney, Acupuncture, Herbal Medicine). Articles were reviewed and summarized.

Results: Herbal medicine has been proven to be free from side-effects and therefore suitable for long term use therapy. Its antilithic beneficial effects include increased urinary volume, increased magnesium excretion (Takusya), inhibitory activity on calcium oxalate aggregation (Takusya, Wulingsan and Desmodyum styracyfolium), inhibition of calcium oxalate nucleation and hydroxyapatite internalization (Wulingsan). In contrast, acupuncture, has shown to be effective as a pre-treatment anxiolytic and analgesic during colic pain and extracorporeal shock wave lithotripsy treatment, reducing the need for complementary sedative drugs.

Conclusion: Chinese traditional medicine is promising as regards its role in stone prevention. An effort must be made in order to standardize study protocols to better assess acupuncture results since each procedure differs in regards to selected acupoints, electrostimulation technique and adjunct anesthetics. Similarly, standardization of Kampo formulations and acceptable clinical endpoints (imaging vs. symptomatic events) is needed.
\end{abstract}

Key words: urolithiasis; medicine, Chinese traditional; medicine, Kampo; acupuncture

Int Braz J Urol. 2009; 35: 396-405

\section{INTRODUCTION}

Urolithiasis is known to be an affliction to mankind from ancient eras (1) and remains a major issue regarding health and well being today. Stone disease affects 10 to $12 \%$ of the population in industrialized countries with a peak incidence between 20 and 40 years of age (2) and is associated with a significant medical expenditure that exceeds 1.5 billion dollars annually in the United
States (3). An additional concern is the high recurrence rate, which is more than $50 \%$ after 10 years (4).

Therefore, great interest has arisen among both physicians and patients towards identifying effective measures to achieve analgesia during renal colic, promote stone passage or stone dissolution and prevent stone recurrence (5). It is of critical importance to consider treatments that may be complementary to conventional Western therapies such as 
Traditional Chinese Medicine (TCM) that have been practiced for over 3000 years (6).

Historically, TCM makes reference to stone disease as "stone urinate". Symptoms of this disease include stone in urine, painful urination, and lower abdominal pain with radiation to the umbilical region (7). According to TCM criteria, renal stone diseases belong to the "sha-lin" (strangury from urolithiasis), "shi-lin" (strangury caused by urinary calculus) and "xue-lin" (strangury complicated by hematuria) (8). TCM treatment encompasses the urinary system and balances the mineral electrolytes as well as the entire endocrine system including prostaglandins and the various neuro-transmitters $(9,10)$. It focuses on reestablishing system balance before stone formation instead of only treating it (6).

We present a literature review of two main fields of Chinese Medicine in stone disease management: Kampo medicine and Acupuncture and the currently existing supporting evidence regarding these types of treatment.

\section{KAMPO MEDICINE}

Kampo medicine is a traditional Japanese herbal therapeutic system that originated in China (Kampo means "medicine from China"). Kampo medicine formed the basis of Chinese and then Japanese medicine for at least 10 centuries until it was superseded by Western medicine around the 1870 s (11). The long-term use of Kampo medicine has been reported to be associated with little or no side effects (12) and therefore seems suitable for long term stone disease treatment in contrast to some oral medications which have shown positive effects but in long term use it is associated with significant drop outs and side effects $(13,14)$.

Theoretically, an effective herbal medicine for stone disease may inhibit nucleation, growth or aggregation of crystals, or might help with expulsion of small crystals through an effect on urothelial adherence or by a diuretic mechanism of action (6).

Kampo medicines are usually a mixture of two or more kinds of herbs, and studies of individual components are uncommon. Its use against kidney stone disease has been described in humans and other animals (15). This review will present the most cited and objectively investigated substances in literature utilized by this system.

\section{Chorei-to}

Chorei-to consists of a mixture of herbs believed to be beneficial for curing nephritis and nephrolithiasis (16). Chorei-to extract has had no inhibitory effect on the formation of amorphous calcium phosphate but did show significant inhibitory effects on the transformation to hydroxyapatite in vitro (16). Another in vitro study analyzed separately two Chorei-to components; Takusya (Alismatis Rhizoma) and Kagosou (Purunellae Spica). Both were proven to strongly inhibit calcium oxalate crystal growth and aggregation in a synthetic urine model (11).

In vivo experiments have shown a weaker effect for Kagosou compared to Takusya. Wistar rats were given $0.5 \mu \mathrm{g}$ ethylene glycol $5 \%$ daily through a feeding tube for 4 weeks. Takusya was able to significantly decrease the tissue calcium concentration in the kidney compared to controls when given in low doses (correspondent to human daily dosage per unit body weight) and reduce calcium oxalate deposits, most notably inside the tubular lumen (11).

Takusya was also shown to reduce tissue calcium oxalate deposits in the kidney of rats treated with stone forming substances ethylene glycol and vitamin $\mathrm{D}_{3}$ (17). Additionally, it has been shown to decrease osteopontin (OPN) expression; the secretion of OPN and its subsequent incorporation into stone matrix may influence the nucleation and aggregative growth of stones (18).

In vivo results suggested that although Chorei-to extract reduced urinary citrate concentration $(11,16,19,20)$ and enhanced calcium excretion $(17,19)$, beneficial changes including increased urinary volume, increased magnesium excretion (19) and potent inhibitory activity on aggregation and kidney deposition identified in Takusya lead to improved supersaturation for calcium oxalate (11). Another in vivo study, however, did not corroborate a diuretic mechanism of action, but also showed that Chorei-to does not promote urinary oxalate excretion as one would expect from tea-like preparations $(19,21)$. 


\section{Wullingsan (WLS)}

Wullingsan is a combination of 5 dried herbs: Alisma orientalis (which provides the Alisma Rhizoma or Takusya, previously mentioned), Polyporus umbellatus, Poria cocos, and Cinnamomon cassia. Indications for WLS administration have varied over the centuries, but originally were used for symptoms of headache, fever, irritability, strong thirst with vomiting immediately after drinking, urinary difficulty and a "floating pulse". Its formula was first mentioned in the TCM book Shang han lun in the third century (7).

WLS has been reported to be a diuretic, and to be able to drain dampness, strengthen the spleen, warm the Yin ("cold nature") and promote Qi transformation $(7,22)$.

WLS properties were assessed both in vitro and in vivo by Vietnamese researchers (22). They evaluated the pharmacological effects of WLS on calcium oxalate nucleation, crystal growth and aggregation. They reported that WLS extract is capable of inhibiting nucleation by prolonging the induction time $(\mathrm{p}<0.001)$ in a dose dependent fashion. WLS also led to inhibition of growth by $28.8 \%$ and $16.46 \%$ in 3.125 and $6.25 \mathrm{mg} / \mathrm{mL}$ respectively, although not statistically significant. At a higher dose $(12.5 \mathrm{mg} / \mathrm{mL})$ it paradoxically promoted the growth of calcium oxalate (CaOx) crystallization by $371 \%$. WLS significantly inhibited $\mathrm{CaOx}$ aggregation in a dose dependent manner (up to $92.49 \%$ at a concentration of $50 \mathrm{mg} / \mathrm{mL}$ WLS extract). In conclusion, WLS extract may inhibit the crystallization of $\mathrm{CaOx}$ by blocking the formation of the crystal nucleus and macrocrystal agglomeration with a potential to prevent stone formation (22).

WLS was tested by Tsai et al. in a SpragueDawley rat model of ethylene glycol-induced (EG) nephrocalcinosis in both low and high dosage and compared to placebo. Histologically, EG-fed rats treated with WLS developed lower crystal deposit scores $(p=0.035)$ [Tsai]. This was also observed in another rat model for nephrocalcinosis where animals were fed with a high phosphorus diet (20). In this study, the individual components failed to provide the same result when tested alone, suggesting a synergy by the presence of complementary components in WLS (16).
In addition to the properties of Takusya discussed in the preceding section, it has been suggested that WLS decreases the concentration of PO4 ions in the fluid in the tubules and inhibits the internalization of hydroxyapatite into the cells $(7,20)$.

\section{Jin Qian Cao (Desmodium styracifolium Merr)}

This herb is believed to act as a diuretic, to promote urine acidification, reduce "heat" and swelling (6). Its extract has been demonstrated in vitro to inhibit crystal growth and aggregation, though it did promote nucleation of crystals. No dissolution of pure monohydrate calcium oxalate crystal mass was observed (6).

Hirayama et al. studied the anti-lithogenic properties of the main component of Desmodium, which is called Desmodium styracyfolium-triterpenoide (Ds- $t$ ) in rats. They used an ethylene glycol and 1-alpha $(\mathrm{OH}) \mathrm{D} 3$ induced oxalate stone animal model. Ds-t fed group demonstrated significantly lower Calcium/Creatinine and Phosphorus/Cr excretion after 3 weeks when compared to control $(\mathrm{p}<0.05)$. Citrate $/ \mathrm{Cr}$ excretion was increased $(p<0.05)$ and so was the 24 hours creatinine clearance $(p<0.01)$. No change in $\mathrm{pH}$ was noted. A $52 \%$ in stone burden was observed in Ds-t fed rats compared to control group animals. This data suggests that Ds-t reduces stone formation of pure $\mathrm{Ca}$ oxalate crystals in the rat kidney (23).

Ds- $t$ is believed to block urinary calcium excretion by either inhibiting the endogenous synthesis of 1-25(OH)2D from 1-alphaD3 in the kidney or by inhibiting the action of $1-25(\mathrm{OH}) 2 \mathrm{D}$ on increased intestinal absorption of $\mathrm{Ca}$. Higher serum calcium levels in Ds-t group also suggests Ca reabsorption in the renal tubules but further studies are needed to clarify this mechanism (23).

\section{Niao Shi Mixture (NSM)}

NSM is a mixture of several different herbs: Squama Manitis, Spina Gleditsiae, Resina Olibani, Resina Myrrhae, Radix Achyranthis Bidentatae, Radix Angelicae Dahuricae, Pericarpium Citri Reticulatae Viride, Semen Coicis, Cortex Magnoliae Officinalis, Fructus Aurantii, Semen Persicae, Rhizoma Sparga- 
nii, Rhizoma Zedoariae, Semen Plantaginis, Radix Paeoniae Rubra and Herba Lysimachiae (24).

For more than 30 years, NSM has been used clinically at Guanganmen Hospital (Beijing, China) to treat urinary calculus disease (24). NSM effects were assessed in four experimental animal models of hyperoxaluric urolithiasis (4-hydroxy-L-proline (HyP), glycoxylic acid, oxalic acid and sodium oxalate). It was quite effective in preventing HyP-induced stones, but had no influence on the other animal models, suggesting its blocking effect to occur prior to the metabolism of HyP glyoxylic acid. NSM was also demonstrated to decrease serum calcium and urinary acid urate although this decrease was not statistically significant (24).

In summary, though in vitro and animal studies suggest a potential therapeutic effect for herbal approaches to stone disease, clinical trials are lacking and as such, claims of effectiveness in humans remains unsubstantiated (Table-1).

\section{ACUPUNCTURE}

\section{Historical Considerations}

Acupuncture is an ancient traditional Chinese method of medical treatment involving the insertion of fine single-use sterile needles in determined points of the body according to a system of channels and meridians developed by early practitioners of TCM over 2000 years ago (25). Needles can be stimulated by manual manipulation, electrical stimulation or heat (26). Approximately 1 million patients visited an acupuncture professional in the United States in 1994 (27).

Its use has been focused on the management of acute and chronic neuromuscular pain, anesthesia for minor operation and pain control in malignant disease (28). It has been proposed as an alternative and effective analgesic with few side effects (29), rapid recovery to work (30) and rare complications (31-34).

Acupuncture inclusion along with other Complementary and Alternative Medicine (CAM) has been increasing in Urology. Many groups such as the Committee of Complementary and Alternative Medicine within the American Urological Association (AUA) recognize the integration of non-conventional therapies into urological clinical practice. Additionally, both public demand for CAM therapies and their testing and validation under modern scientific research patterns methodology has increased $(35,36)$. In the literature, acupuncture has been described to aid in the management of chronic prostatitis, chronic pelvic pain as well as nocturnal enuresis $(29,36-38)$.

Table 1 - Herbal medicines (Kampo) overview.

\begin{tabular}{|c|c|c|}
\hline Herb & Mechanism of Action & Study Type \\
\hline \multirow[t]{4}{*}{ Chorei-to } & Inhibits calcium phosphate transformation into hydroxyapatite & In vitro \\
\hline & Inhibits calcium oxalation, aggregation and crystal growth & In vitro \\
\hline & $\begin{array}{l}\text { Decreases tissue calcium concentration in kidney and calcium } \\
\text { oxalate deposits in tubular lumen }\end{array}$ & In vivo (Rat) \\
\hline & $\begin{array}{l}\text { Improves supersaturation for calcium oxalate } \\
\text { (increases urine volume, magnesium excretion) }\end{array}$ & In vivo (Rat) \\
\hline \multirow[t]{2}{*}{ Wullingsan } & Inhibits calcium oxalate nucleation, crystal growth and aggregation & In vitro and in vivo (Rat) \\
\hline & Lower crystal deposits in nephrocalcinosis rat model & In vivo (Rat) \\
\hline Jin Qian Cao & $\begin{array}{l}\text { Lower calcium excretion and increases urine citrate and 24-hours } \\
\text { creatinine excretion in oxalate stone model }\end{array}$ & In vivo (Rat) \\
\hline \multirow[t]{2}{*}{ Niao Shi Mixture } & Prevents 4-hydroxy-L-proline (HyP) induced stones & In vivo (Rat) \\
\hline & Decreases serum calcium and urate acid & In vivo (Rat) \\
\hline
\end{tabular}


In stone disease field, acupuncture has been used for treating renal colic (28) and for extracorporeal shock wave lithotripsy (SWL) - related pain and anxiety $(30,37,39-42)$.

\section{Supporting Objective Evidence}

Lee et al. conducted a prospective randomized study to compare the effect of acupuncture and intramuscular analgesic Avafortan ${ }^{\mathrm{TM}}$ (Asta-Werke Degussa PharmaGruppe, West Germany) in the treatment of renal colic. Acupuncture was applied in 4 to 6 points in the hand (loin and leg, EX-UE 7) or on the back (points UB-21 through UB-25, UB-45 through UB47 or Yao-Yen EX-B7) according to the main site of pain and electrically stimulated $(3 \mathrm{~Hz})$ in more than half of the cases $(68.2 \%)$. No statistically significant difference was noted regarding pain scores. Onset of analgesic effect occurred more rapidly in acupuncture patients than for Avafortan ${ }^{\mathrm{TM}}(3.1 \pm 2.9$ vs. $15.4 \pm 7.6$ minutes; $p<0.05)$. Acupuncture had no reported side effects. Forty-three percent of patients in Avafortan ${ }^{\mathrm{TM}}$ group reported minor side effects, such as skin rash, drowsiness, tachycardia and facial flush. Neither treatment had any immediate effect on stone location. The authors emphasized that acupuncture may be particularly helpful in some selected situations such as drug allergy or fear of drug addiction, or pregnancy (28).

Mora et al. evaluated the use of auricular acupressure as a treatment for anxiety in elderly individuals who are transported by ambulance before receiving SWL in an Italian medical facility. One hundred patients were prospectively enrolled in a randomized, double-blind sham-controlled study. A self-assessment Visual Analog Scale (VAS) was completed before and after intervention and rated the level of anxiety experienced by each individual. Auricular acupressure (AA) was administered with a plastic ball $1 \mathrm{~mm}$ in diameter, which was pressed on the relaxation point and fixed with an ear patch. This point is located at the superior lateral wall of the triangular fossa [41]. The sham group received AA at a sham point with no relaxing or anxiolytic effect in acupuncture (39). Individuals who received acupressure at the relaxation point had significantly decreased anxiety and pain scores and were also more optimistic towards their treatment upon arrival at the hospital than individuals who underwent sham intervention ( $p$ $=0.001$ ). Therefore, acupressure proved to be a useful measure for decreasing anxiety in the pre-interventional urological setting. The authors proposed that minimal equipment and training is required, making it a technique applicable to any physician, nurse, paramedic or medical technician (40).

Similarly, Wang et al. conducted a doubleblind randomized sham-controlled study to evaluate acupuncture use as an adjunct for the preprocedural anxiety and pain management in 48 patients undergoing lithotripsy procedures. Patients completed a baseline State-Trait Anxiety Inventory (STAI), which is a 40-item self-report measure of anxiety status. An experienced acupuncturist performed auricular acupuncture intervention ipsilateral to patient's dominant hand at the Relaxation, Master Cerebral, and Valium points of the Ear and patients in the sham group received auricular acupuncture at the wrist, shoulder, and extra-auricular sham points of the ear. Thirty minutes after initiation of the acupuncture procedure, all patients completed a second STAI. Patients were then sent to SWL procedure, before which they received a pre-anesthetic cocktail containing midazolam, alfentanil and metoclopramide. During the procedure, they could also self-administer alfentanil through a patient-controlled analgesia (PCA) pump as needed.

During the SWL treatment, the Acupuncture group received body acupuncture at bilateral Liver 3 and Large Intestine 4 points. Liver 3 is located on the dorsum of the foot in the depression distal to the junctions of the first and second metatarsals, and Large Intestinal 4 is located on the radial side of the middle of the second metacarpal (38). Needles were electrically stimulated under $2 \mathrm{~Hz}$ and $25 \mathrm{~V}$. Sham group received only a very superficial insertion (1-2 mm deep). Standardized SWL procedure was carried out, using 2500 shocks for renal calculi and 3000 shocks for ureteral calculi.

Alfentanil consumption was significantly lower in Acupuncture Group ( $\mathrm{p}=0.040)$. Pain scores assessed by VAS and at discharge (assessed by shortform McGill questionnaires) (43) were also significantly lower ( $\mathrm{p}=0.014$ and 0.047 respectively). The authors concluded that a combination of auricular and body acupuncture decrease preprocedural anxiety 
and intraprocedural alfentanil requirements during lithotripsy (38).

Rogenhofer et al. also evaluated the role of acupuncture for pain control during SWL. Their nonrandomized study prospectively enrolled 90 patients with a median age of 51.7 years. They received acupuncture immediately prior to ESWL treatment. Needles were inserted 1 to $2 \mathrm{~cm}$ deep until the "De Qi", a feeling of numbness and tingling within the range of the acupoint, was achieved. Acupoints comprised a total of 9 traditional points generally considered either for analgesia, especially of the lower back (Large Intestine 4, Governing Vessel 20, Urinary Bladder 23, Urinary Bladder 52, Kidney 3 ) or for anxiety related disorders (Spleen 6). Additional analgesic medication was given as needed to address break-through pain (piritramide and midazolam). ESWL was performed using a Siemens Lithostar lithotripter, (Erlanger, Germany) in a standardized fashion (renal stones and stones in proximal ureter received 4000 shocks at a maximum of $18.2 \mathrm{kV}$; middle and distal ureteral stones received 5000 impulses of up to $21 \mathrm{kV}$ ). Ninety-three percent of patients expressed willingness to undergo any necessary repeated ESWL under acupuncture analgesia; and $87.5 \%$ considered their pain control to be good or very good. Average pain intensity reported was similar to the ones described for drug combination (1.5 to 2.5 on a 10 - point VAS) (41).

In small case series, Quatan et al. also reported success in treating three SWL patients with acupuncture, who had not tolerated conventional analgesic medications (acetaminophen, piroxicam, midazolam, pethidine). Acupuncture was performed half an hour prior to commencing ESWL and needles underwent electrical stimulation using a pulse generator set to $4 \mathrm{~Hz}$. Acupoints were CV4, ST25, BL23 and BL25 (37). They reported satisfactory analgesia and no side effects concluding that acupuncture should be offered to people who would not otherwise be suitable for outpatient SWL services or those who request it (37).

Finally, Karamarz et al. performed a prospective, randomized, sham-controlled study to evaluate the efficacy of transcutaneous electrical nerve stimulation (TENS) versus conventional-like acupuncture as an adjunct for analgesia in patients undergoing SWL treatment. Each group comprised 22 patients. A $2 \mathrm{~Hz}$ stimulation frequency was used in acupuncture and control groups with a difference in shock intensity above $15 \mathrm{~mA}$ and below $10 \mathrm{~mA}$, respectively. Eighty hertz were used in TENS group. Shockwave energy was estimated by an index described by Schelling et al., which considers both voltage and number of shocks (44). During the operation, patients were oriented to utilize the PCA button whenever the VAS > 3 . VAS pain scores and alfentanil consumption were lower in TENS group comparing to the other two groups $(\mathrm{p}<0.001$ and $\mathrm{p}<0.05)$. The authors suggest conventional TENS use during ESWL to shorten recovery time and reduce alfentanil consumption and side-effects (42).

Resim et al. performed a similar prospective, randomized study of 35 patients, comparing electroacupuncture (EA) to conventional anesthetic agents during SWL. Midazolam $\left(1.5 \mathrm{mg} / \mathrm{KG}_{\mathrm{G}}\right)$ and tramadol $(0.06 \mathrm{mg} / \mathrm{Kg})$ were used for sedation and analgesia versus electroacupuncture delivered to urinary bladder points 20,21,22,23 and 52. Needles were stimulated manually until the patient felt the sensation as Qi numbness in that region. Electrical stimulation with 2-4 Hz frequency with an intensity up to the tolerance level of each patient was given. Achieved energy levels were statistically comparable between the two groups, although SWL protocols were not standardized. VAS scores were consistently lower in the EA group, but with no statistical significance $(p=0.245)$. No difference in stone-free rates was found either. Both treatment regimens offered satisfactory sedation and analgesia and allowed the maximum number shockwaves to be given. No side effects were seen in any patients who received EA treatment. Therefore, EA was proven to be an effective alternative to other forms of anesthesia and analgesia during SWL procedures (30).

\section{Mechanisms of Action}

Acupuncture is believed to stimulate humoral factors and neurological pathways through a specific point with a needle or with pressure which activates small myelinated nerve fibers that send impulses into the spinal cord, mid brain, pituitary gland and hypothalamus (45), causing a measurable amount of endorphins to be released into the blood (46). Further- 
Table 2 - Acupuncture article reviews.

\begin{tabular}{|c|c|c|c|c|}
\hline Author & Study Design & Conclusion & $\begin{array}{l}\text { Level of } \\
\text { Evidence }\end{array}$ & $\begin{array}{c}\text { Grade of } \\
\text { Recommendation }\end{array}$ \\
\hline Wang et al. (2001) & $\begin{array}{l}\text { RCT with } 48 \\
\text { pts undergoing } \\
\text { SWL }\end{array}$ & $\begin{array}{c}\text { Acupuncture decreases preprocedural } \\
\text { anxiety and alfentanil requirement } \\
\text { during SWL }\end{array}$ & $1 b$ & A \\
\hline Karamarz et al. (2004) & $\begin{array}{l}\text { RCT with } 44 \\
\text { pts undergoing } \\
\text { SWL }\end{array}$ & $\begin{array}{l}\text { TENS reduces recovery time and } \\
\text { alfentanil requirement during SWL } \\
\text { compared to acupuncture }\end{array}$ & $1 b$ & A \\
\hline Mora et al. (2007) & $\begin{array}{l}\text { RCT with } 100 \\
\text { pts undergoing } \\
\text { SWL }\end{array}$ & $\begin{array}{l}\text { Auricular acupuncture decreases } \\
\text { anxiety in the preinterventional urolo- } \\
\text { gical setting }\end{array}$ & $2 b$ & B \\
\hline Lee et al. (1992) & $\begin{array}{l}\text { RCT with } 38 \\
\text { pts for renal } \\
\text { colic }\end{array}$ & $\begin{array}{l}\text { Same pain score, analgesia onset } \\
\text { more rapid with Avafortan than acu- } \\
\text { puncture }\end{array}$ & $3 b$ & B \\
\hline Quatan et al. (2003) & $\begin{array}{l}\text { Case series of } \\
3 \text { pts for SWL }\end{array}$ & $\begin{array}{l}\text { Satisfying analgesia, should be an } \\
\text { option for pts intolerant to conventio- } \\
\text { nal analgesics }\end{array}$ & 4 & $\mathrm{C}$ \\
\hline Rogenhofer et al. (2004) & $\begin{array}{l}\text { Case series of } \\
90 \text { pts for SWL }\end{array}$ & $\begin{array}{l}\text { Satisfying analgesia, no } \\
\text { side-effects }\end{array}$ & 4 & $\mathrm{C}$ \\
\hline
\end{tabular}

1. Canadian Task Force on the Periodic Health Examination: The periodic health examination. CMAJ 1979; 121: 1193-254.

2. Sackett DL: Rules of evidence and clinical recommendations on use of antithrombotic agents. Chest. 1986; 89 (2 suppl): 2S-3S.

3. Cook DJ, Guyatt GH, Laupacis A, Sackett DL, Goldberg RJ: Clinical recommendations using levels of evidence for antithrombotic agents. Chest. 1995; 108 (4 Suppl): 227S-230S.

4. Yusuf S, Cairns JA, Camm AJ, Fallen EL, Gersh BJ. Evidence-Based Cardiology. London: BMJ Publishing Group. 1998.

more, various neurotransmitters such as serotonin, norepinephrine and possibly gamma-amynobutyric acid are known to interrupt incoming stress signals in the central nervous system.

Several studies have confirmed the ability of acupuncture to induce opiate-like peptides (beta-endorphin, dynorphin and met-enkephalin) in the cerebrospinal fluid and to provide pain relief (28). The humoral mechanism can be inhibited by naloxone (47).

Neural pathways can be addressed through segmental and nonsegmental routes. Segmental acupuncture analgesia is produced by short circuits and non segmental acupuncture analgesia is produced by afferent signals that synapse on spinal interneurons and then project to higher brain centers through the anterolateral tract $(48,49)$.

The addition of electrical stimulation during acupuncture induces endorphin release that generates a more potent effect on the concentration of neuropep- tides in the animal hippocampus and occipital cortex than manual acupuncture (50) which can be noted as an enhancement in signal magnitude in MRI imaging. An autonomic response also occurs as evidenced by significant reduction in average heart rate during treatment (51). Some studies also suggest that acupuncture treatment can decrease inflammation and relieve pain $(52,53)$.

Locally, intramuscular movements of the needle cause insertional activity, or depolarization of innervated single or grouped muscle fibers. These are micro-twitches that produce micro-stretch effects on the muscle fibers nearby, thus reducing the local pressure on pain-sensitive structures such as intramuscular nerves and blood vessels (54).

The effect of acupuncture as analgesia depends also on the placebo effect and suggestion effect ("physician as a drug") that takes place in every treatment and medication (41). 


\section{CONCLUSION}

Chinese Traditional Medicine holds promise for its role in stone prevention through the use of Kampo herbal compound, though no evidence of dissolution properties exist. Acupuncture has been demonstrated to be an effective adjunct in stone disease treatment both as an analgesic during renal colic or SWL therapy and for reducing pre-treatment anxiety (Table-2).

An effort must be made in order to standardize study protocols to better assess acupuncture results since each one differs in regards to selected acupoints, electrostimulation technique and adjunct anesthetics. Similarly, standardization of Kampo formulations and acceptable clinical endpoints (imaging vs. symptomatic events) are needed.

\section{CONFLICT OF INTEREST}

Dr. Ricardo Miyaoka is corporate sponsored endourology Fellow with Gyrus ACMI / Olympus.

\section{REFERENCES}

1. Cledeling, L: Source Book of Medical History. New York, Dove Publications. 1942; pp. 14-8.

2. Pak CYC: Citrate and renal calculi. Miner Electrol Metab. 1987; 13: 257-66.

3. Schwartz BF, Schenkman N, Nguyen R, Stoller ML: Gahat: a Napalese cure for urolithiasis? Urology. 2000; 56: 912-4.

4. Wasserstein AG: Nephrolithiasis: acute management and prevention. Dis Mon. 1998; 44: 196-213.

5. Kieley S, Dwivedi R, Monga M: Ayurvedic medicine and renal calculi. J Endourol. 2008; 22: 1613-6.

6. Gohel MD, Wong SP: Chinese herbal medicines and their efficacy in treating renal stones. Urol Res. 2006; 34: 365-72.

7. Tsai CH, Chen YC, Chen LD, Pan TC, Ho CY, Lai MT, et al.: A traditional Chinese herbal antilithic formula, Wulingsan, effectively prevents the renal deposition of calcium oxalate crystal in ethylene glycol-fed rats. Urol Res. 2008; 36: 17-24.

8. The Traditional Chinese Medicine (TCM) [Internet]. [cited 4 Sept 2006] Available from: http://www.tcmtreatment.com
9. Zhao Z: An illustrated Chinese Materia Medica in Hong Kong School of Chinese Medicine, Hong Kong Baptist University, Hong Kong. 2004.

10. 10. Chen JK, Chen TT: Chinese Medical Herbology and Pharmacology. The Art of Medicine. California, Press Inc. 2004.

11. Koide T, Yamaguchi S, Utsunomiya M, Yoshioka T, Sugiyama K: The inhibitory effect of kampou extracts on in vitro calcium oxalate crystallization and in vivo stone formation in an animal model. Int J Urol. 1995; 2: 81-6.

12. Product Catalogue. Tsumura Kampo Medicine Extract Granules for Ethical Use. Tokyo, Tsumura Co. Ltd. 1990.

13. Gürocak S, Küpeli B: Consumption of historical and current phytotherapeutic agents for urolithiasis: a critical review. J Urol. 2006; 176: 450-5.

14. Coe FL, Evan A, Worcester E: Kidney stone disease. J Clin Invest. 2005; 115: 2598-608.

15. Sugimoto K, Sakurai N, Shirasawa H, Fujise Y, Shibata K, Shimoda K, et al.: Bovine cases of urolithiasis treated with traditional herbal medicine, P-3. J Vet Med Sci. 1992; 54: 579-82.

16. Hidaka S, Abe K, Liu SY: A new method for the study of the formation and transformation of calcium phosphate precipitates: effects of several chemical agents and Chinese folk medicines. Arch Oral Biol. 1991; 36: 49-54.

17. Yasui T, Fujita K, Sato M, Sugimoto M, Iguchi M, Nomura S, et al.: The effect of takusha, a kampo medicine, on renal stone formation and osteopontin expression in a rat urolithiasis model. Urol Res. 1999; 27: 194-9.

18. McKee MD, Nanci A, Khan SR: Ultrastructural immunodetection of osteopontin and osteocalcin as major matrix components of renal calculi. J Bone Miner Res. 1995; 10: 1913-29.

19. Ogawa Y, Takahashi S, Kitagawa R: Effect of herb medicines for urolithiasis on urinary oxalate excretion in rats. Hinyokika Kiyo. 1983; 29: 1269-71.

20. Liu QL, Sato S, Kishikawa T, Matsuzaki H, Yamanaka $\mathrm{N}$ : Effectiveness of a traditional Chinese medicine, Wulingsan, in suppressing the development of nephrocalcinosis induced by a high phosphorus diet in young rats. Med Electron Microsc. 2001; 34: 103-14.

21. McKay DW, Seviour JP, Comerford A, Vasdev S, Massey LK: Herbal tea: an alternative to regular tea for those who form calcium oxalate stones. J Am Diet Assoc. 1995; 95: 360-1.

22. Chen YC, Ho CY, Chen LD, Hsu SF, Chen WC: Wu-Ling-San formula inhibits the crystallization of 
calcium oxalate in vitro. Am J Chin Med. 2007; 35: 533-41.

23. Hirayama H, Wang Z, Nishi K, Ogawa A, Ishimatu T, Ueda S, et al.: Effect of Desmodium styracifoliumtriterpenoid on calcium oxalate renal stones. Br J Urol. 1993; 71: 143-7.

24. Xue JZ: A preliminary laboratory investigation of "niao shi mixture" in preventing experimental calcium oxalate renal calculus in rats. J Tradit Chin Med. 1982; 2: 187-94.

25. Capodice JL, Bemis DL, Buttyan R, Kaplan SA, Katz AE: Complementary and alternative medicine for chronic prostatitis/chronic pelvic pain syndrome. Evid Based Complement Alternat Med. 2005; 2: 495-501.

26. Ellis A, Wiseman N, Boss K: Fundamentals of Chinese Acupuncture Brookline. Massachusetts, Paradigm Publications. 1991.

27. Paramore LC: Use of alternative therapies: estimates from the 1994 Robert Wood Johnson Foundation National Access to Care Survey. J Pain Symptom Manage. 1997; 13: 83-9.

28. Lee YH, Lee WC, Chen MT, Huang JK, Chung C, Chang LS: Acupuncture in the treatment of renal colic. J Urol. 1992; 147: 16-8.

29. Chen R, Nickel JC: Acupuncture ameliorates symptoms in men with chronic prostatitis/chronic pelvic pain syndrome. Urology. 2003; 61: 1156-9; discussion 1159.

30. Resim S, Gumusalan Y, Ekerbicer HC, Sahin MA, Sahinkanat T: Effectiveness of electro-acupuncture compared to sedo-analgesics in relieving pain during shockwave lithotripsy. Urol Res. 2005; 33: 285-90.

31. Matsuyama H, Nagao K, Yamakawa GI, Akahoshi K, Naito K: Retroperitoneal hematoma due to rupture of a pseudoaneurysm caused by acupuncture therapy. J Urol. 1998; 159: 2087-8.

32. Fujiwara T, Tanohata K, Nagase M: Pseudoaneurysm caused by acupuncture: a rare complication. AJR Am J Roentgenol. 1994; 162: 731.

33. Halvorsen TB, Anda SS, Naess AB, Levang OW: Fatal cardiac tamponade after acupuncture through congenital sternal foramen. Lancet. 1995; 345: 1175.

34. Carron H, Epstein BS, Grand B: Complications of acupuncture. JAMA. 1974; 228: 1552-4.

35. Hirsch IH: Integrative urology: a spectrum of complementary and alternative therapy. Urology. 2000; 56: 185-9.

36. Capodice JL, Jin Z, Bemis DL, Samadi D, Stone BA, Kapan S, et al.: A pilot study on acupuncture for lower urinary tract symptoms related to chronic prostatitis/ chronic pelvic pain. Chin Med. 2007; 2: 1.
37. Quatan N, Bailey C, Larking A, Boyd PJ, Watkin N: Sticks and stones: use of acupuncture in extracorporeal shockwave lithotripsy. J Endourol. 2003; 17: 867-70.

38. Wang SM, Kain ZN: Auricular acupuncture: a potential treatment for anxiety. Anesth Analg. 2001; 92: 548-53.

39. Wang SM, Punjala M, Weiss D, Anderson K, Kain ZN: Acupuncture as an adjunct for sedation during lithotripsy. J Altern Complement Med. 2007; 13: 241-6.

40. Mora B, Iannuzzi M, Lang T, Steinlechner B, Barker R, Dobrovits M, et al.: Auricular acupressure as a treatment for anxiety before extracorporeal shock wave lithotripsy in the elderly. J Urol. 2007; 178: 160-4; discussion 164.

41. Rogenhofer S, Wimmer K, Blana A, Roessler W, Wieland WF, Filbeck T: Acupuncture for pain in extracorporeal shockwave lithotripsy. J Endourol. 2004; 18: 634-7.

42. Kararmaz A, Kaya S, Karaman H, Turhanoglu S: Effect of the frequency of transcutaneous electrical nerve stimulation on analgesia during extracorporeal shock wave lithotripsy. Urol Res. 2004; 32: 411-5.

43. Melzack R: The short-form McGill Pain Questionnaire. Pain. 1987; 30: 191-7.

44. Schelling G, Mendl G, Weber W, Pauletzki J, Sackmann M, Pöppel E, et al.: Patient controlled analgesia for extracorporeal shock wave lithotripsy of gallstones. Pain. 1992; 48: 355-9.

45. Hui KK, Liu J, Makris N, Gollub RL, Chen AJ, Moore CI, et al.: Acupuncture modulates the limbic system and subcortical gray structures of the human brain: evidence from fMRI studies in normal subjects. Hum Brain Mapp. 2000; 9: 13-25.

46. Levine JD, Gormley J, Fields HL: Observations on the analgesic effects of needle puncture (acupuncture). Pain. 1976; 2: 149-59.

47. Mayer DJ, Price DD, Rafii A: Antagonism of acupuncture analgesia in man by the narcotic antagonist naloxone. Brain Res. 1977; 121: 368-72.

48. Klarskov P: Enkephalin inhibits presynaptically the contractility of urinary tract smooth muscle. Br J Urol. 1987; 59: 31-5.

49. Pomeranz B: Neural mechanisms of acupuncture analgesia. In: Persistent Pain (ed.), Moderrn Methods of Treatment. New York, Lipton and J. Miles. Academic Press. 1981; pp. 241-52.

50. Bucinskaite V, Lundeberg T, Stenfors C, Ekblom A, Dahlin L, Theodorsson E: Effects of electro-acupuncture and physical exercise on regional concentrations of neuropeptides in rat brain. Brain Res. 1994; 666: 128-32.

51. Wu MT, Hsieh JC, Xiong J, Yang CF, Pan HB, Chen $\mathrm{YC}$, et al.: Central nervous pathway for acupuncture 
stimulation: localization of processing with functional MR imaging of the brain--preliminary experience. Radiology. 1999; 212: 133-41.

52. Watkin H: Back pain--an integrated approach in primary care. Acupunct Med. 2004; 22: 203-6.

53. Sekido R, Ishimaru K, Sakita M: Differences of electroacupuncture-induced analgesic effect in normal

\author{
Correspondence address: \\ Dr. Ricardo Miyaoka \\ Department of Urologic Surgery \\ University of Minnesota \\ 1420 Delaware St. SE (MMC 394) \\ Minneapolis MN 55455, USA \\ Fax: + 1612 626-0428
}

\section{EDITORIAL COMMENT}

Dietary measures and pharmacologic therapy constitute the mainstay of stone metaphylactic programs. Despite proven efficacy in preventing stone recurrence, however, compliance with drug and dietary therapy historically remains poor because of cost, side effects, inconvenience and lifestyle changes necessitated by incorporating these regimens. An effective, low cost, well-tolerated alternative therapy might improve compliance and reduce the morbidity of stone recurrence. Herbal medicines, particularly traditional Chinese medicines, have been promoted as potential alternatives to established treatments in preventing stone formation. Miyaoka and Monga offer a comprehensive and balanced review of published scientific studies that address the effectiveness of traditional Chinese medicines in reducing stoneforming potential. While some of these agents have demonstrated promising effects in vitro or in stoneproducing animal models with regard to inhibiting specific steps in the crystallization or stone forming process, no clinical trials support the benefit of these medicines in reducing actual stone recurrence, and further study is necessary before they can be widely recommended.

Like medical therapy for stone prevention, effective treatments to alleviate the pain of renal colic and inflammatory conditions in rats. Am J Chin Med. 2003; 31: 955-65.

54. Chu J: The local mechanism of acupuncture. Zhonghua Yi Xue Za Zhi (Taipei). 2002; 65: 299-302.

Accepted after revision: April 6, 2009 and the discomfort of shock wave lithotripsy are also readily available with narcotic analgesics and nonsteroidal anti-inflammatory agents. However, these medications too are associated with side effects, such as nausea, constipation, gastrointestinal effects and cognitive dysfunction that in some cases counterbalance the benefit of pain control. Some alternative therapies, such as acupuncture and transcutaneous electrical nerve stimulation reviewed here, have been subjected to randomized, controlled trials that have confirmed their effectiveness in alleviating renal pain. While these treatments have not become mainstream, primarily due to lack of experience or exposure for most physicians, it behooves practitioners to keep an open mind and consider these measures as options, perhaps at least in patients not effectively treated with traditional measures.

Dr. Margaret S. Pearle Department of Urology University of Texas Southwestern Medical Center Dallas, Texas, USA E-mail: margaret.pearle@utsouthwestern.edu 